\title{
THE EXTENSION OF MEASURABLE FUNCTIONS
}

\author{
R. M. SHORTT
}

\begin{abstract}
Say a measurable space $(Y,(B)$ ) has the extension property (resp. the extension property in the restricted sense) if for every measurable space $(X, \widehat{S})$ and every subset $A$ of $X$ (resp. subset $A$ of $X$ with $X \backslash A$ singleton), each function $f$ : $A \rightarrow Y$ measurable for $S(A)=\langle B \cap A: B \in S\rangle$ may be extended to a measurable function $g: X \rightarrow Y$. A countably generated and separated $(Y, U B)$ has the extension property if and only if it is a standard space, i.c. it is isomorphic to a Borel subset of the real line. The discrete space $\left(Y, 2^{Y}\right)$ has the extension property in the restricted sense if and only if the cardinality of $Y$ is not two-valued measurable.
\end{abstract}

We shall use the following notation and terminology: if $(X, \widehat{s})$ is a measurable space, and $A \subset X$, let $\varsigma(A)=\{B \cap A: B \in \Omega\}$. A measurable space $(X, 6)$ is separable if $Q B$ is countably generated and separated (contains singletons); $(X, \because\})$ is standard if there is a complete separable (i.e. Polish) metric on $X$ for which $B$ becomes the associated Borel $\sigma$-algebra; equivalently, $(X, B)$ is isomorphic with some Borel subset of the real numbers.

The extension problem. Let there be given

(1) measurable spaces $(X, S)$ and $\left(Y, \frac{6}{b}\right)$.

(2) a subset $A$ of $X$, and

(3) a function $f: A \rightarrow Y$, measurable from $S(A)$ to $: h$.

We ask whether there is an S-measurable $g: X \rightarrow Y$ extending $f$ (i.e. $g(a)=f(a)$ for $a \in A)$. Say that a measurable space $(Y, \mathscr{B})$ has the extension property if such an extension exists for each $(X, \S), A$ and $f$ as above. If an extension exists whenever $X \backslash A$ is a singleton set, then $(Y, \mathscr{B})$ has the extension property in the restricted sense.

What seem to have been the first positive results for this problem were obtained by von Alexits [1] and Sierpiński [8] in the case of real functions on subsets of Polish spaces; here, particular attention was paid to the Baire class of $f$ and $g$. It is a small step from their results to the following characterization of separable spaces with the extension property:

THEOREM 1. Among separable spaces, those $(Y, 93)$ having the extension property are precisely the standard spaces.

Proof. To prove the extension property for standard spaces, obtain an extension for functions assuming finitely many values and pass to the limit (cf. exercise 13, p. 260 of Cohn [2]); alternatively, derive the result from 2.3 Lemma 1 of Lehmann [4].

Received by the editors November 24, 1981.

1980 Mathematics Subject Classification. Primary 28A20: Secondary 03E15. 03E55.

Key words and phrases. Measurable space, extension property, separable space, standard space, measurable cardinal, two-valued measurable cardinal, separability character. 
For the converse, the well-known method of Marczewski [5] and the separability of $(X, \mathscr{B})$ allow us to consider $X$ as a subset of the unit interval $[0,1]$ with $\mathscr{B}=\mathscr{Q}(X)$ and $Q$ the Borel $\sigma$-algebra of $[0,1]$. Let $f: X \rightarrow X$ be the identity function. If now $f$ has an $Q$-measurable extension $g: I \rightarrow X$, then $X=\{x: g(x)=x\}$ is a Borel subset of $[0,1]$. Thus $(X, \Re)$ is a standard space. Q.E.D.

Let $Y$ be a nonempty set and let $d$ be the metric on $Y$ defined by the rule $d(x, y)=1$ for $x \neq y$ and $d(x, x)=0$. Then $(Y, d)$ becomes a complete metric space under the discrete topology and has the power set $2^{Y}$ as its Borel $\sigma$-algebra. We have not solved the problem of extensions in the case where the "target" space $Y$ is complete, but not separable. For example, we do not know if $\left(\mathbf{R}, 2^{\mathbf{R}}\right)$ has the extension property, where $\mathbf{R}$ is the real line.

Recall that a cardinal number $k$ is measurable if whenever a set $X$ has cardinality $k$, there is a measure $\mu$ on $\left(X, 2^{X}\right)$ such that $\mu\{x\}=0$ for all $x \in X$ and $\mu X=1 ; k$ is two-valued measurable if in addition $\mu$ may be chosen so as to assume only the values 0 and 1 .

According to Ulam [9,p. 146], two-valued measurable cardinals must be extremely large (strongly inaccessible); still larger according to A. Tarski [3]. D. Scott [7] showed that Gödel's axiom of constructibility $V=L$ implies nonexistence of any two-valued measurable cardinals. Apparently it is as yet an open problem whether it is consistent with Zermelo-Fraenkel set theory that any two-valued measurable cardinals exist. In any case the next result shows that essentially all measurable spaces of the form $\left(X, 2^{X}\right)$ have the extension property in the restricted sense.

THEOREM 2. Let $X$ be a set with cardinality $k>0$. Then $\left(X, 2^{X}\right)$ has the extension property in the restricted sense if and only if $k$ is not two-valued measurable.

Proof. Suppose that $k$ is two-valued measurable and let $\mu$ be a nontrivial 0 -1 measure on $\left(X, 2^{X}\right)$. Define $Y=X \cup\{p\}$, where $p$ is a new point $p \notin X$ and set $\mathcal{\delta}=\{B \cup\{p\}: B \subset X, \mu B=1\} \cup\{B: B \subset X, \mu B=0\}$; then $\delta(X)=2^{X}$, and the identity function $f: X \rightarrow X$ is $\delta(X)$-measurable. Suppose that $g: Y \rightarrow X$ is an $\mathcal{S}$-measurable extension of $f$; then $g^{-1}(g(p))=\{p, g(p)\} \notin \delta$, a contradiction.

Assume now that $(Y, \mathcal{S})$ is a measurable space with $A \subset Y$ and $Y \backslash A=\{p\}$ and suppose $f$ is a measurable function from $(A, \mathcal{S}(A))$ to $\left(X, 2^{X}\right)$ with no $\delta$-measurable extension $g: Y \rightarrow X$. Then $\{p\} \notin \mathcal{S}$ and for each $B \in \mathcal{S}(A)$, either $B \cup\{p\} \in \mathcal{S}$ or $B \in \mathcal{S}$, but not both. Define a measure $\mu$ on $(A, \mathcal{S}(A))$ by the rule $\mu B=1$ if $B \cup\{p\} \in \mathcal{S}$ and $\mu B=0$ if $B \in \mathcal{S}$. Then $f(\mu)$ is a $0-1$ measure on $\left(X, 2^{X}\right)$ with $f(\mu)(X)=1$; furthermore, $f(\mu)\{x\}=0$ : if not, then there is an $x \in X$ with $f(\mu)\{x\}$ $=\mu f^{-1}(x)=1$; then $g(p)=x$ defines an $\delta$-measurable extension of $f:$ if $S \subset X$, $x \in S$, then $g^{-1}(S)=f^{-1}(S) \cup\{p\}$ and $\mu f^{-1}(S)=1$, so that $f^{-1}(S) \cup\{p\} \in \Omega$; if $x \notin S$, then $g^{-1}(S)=f^{-1}(S)$ and $\mu f^{-1}(S)=0$, so that $f^{-1}(S) \in \S$. Since no such extension exists, $f(\mu)$ is a nontrivial 0 -1 measure on $\left(X, 2^{X}\right)$ and the cardinality of $X$ is two-valued measurable. Q.E.D.

Since measurable cardinals are weakly inaccessible [9], the continuum hypothesis implies that the cardinal of the continuum is not measurable, and the generalized continuum hypothesis implies that measurable cardinals are strongly inaccessible 
and hence extraordinarily large. So one can assume that cardinalities of spaces arising in analysis are all nonmeasurable.

The separability character of a metric space $X$ is the smallest cardinality of a base for the topology of $X$. A limited type of extension is possible for complete spaces with nonmeasurable separability character.

TheOREM 3. Let $(Y, \mathcal{S}, P)$ be a finite measure space, $A \subset Y$ and $f: A \rightarrow X$ a Borel measurable function from $(A, \delta(A))$ to a complete metric space $X$ with nonmeasurable separability character. Then there is an $\delta$-measurable $g: Y \rightarrow X$ such that

$$
P^{*}\{x \in A: f(x) \neq g(x)\}=0 .
$$

Proof. Consider $P^{*}$ as a measure on $(A, \delta(A))$ and its image $f\left(P^{*}\right)$ on $X$. By a theorem of Marczewski and Sikorski [6, Theorem III], there is a closed subset $F$ of $X$ which is separable and is such that $f\left(P^{*}\right)(F)=f\left(P^{*}\right)(X)=P^{*}(A)$. Let $f_{0}$ be $f$ restricted to $f^{-1}(F)$ with range $F$ and use Theorem 1 to extend $f_{0}$ to an $\delta$-measurable $g: Y \rightarrow F$. Then $\{x \in A: f(x) \neq g(x)\} \subset A \backslash f^{-1}(F)$, which has $P^{*}$-measure $P^{*}(A)$ $P^{*} f^{-1}(F)=P^{*}(A)-f\left(P^{*}\right)(F)=0$. Q.E.D.

Acknowledgments are due R. M. Dudley, who suggested the problem and inspired the subsequent research, also to D. Cohn and D. Allinger for their helpful remarks.

\section{REFERENCES}

1. G. von Alexits, Üher die Erweiterung einer Baireschen Funktion, Fund. Math. 15 (1930), 51-56.

2. D. L. Cohn, Measure theory, Birkhäuser, Basel, 1980.

3. H. J. Keisler and A. Tarski, From accessible to inaccessible cardinals, Fund. Math. 53 (1964). 225-308.

4. E. L. Lehmann, Testing statistical hypotheses, Wiley, New York, 1959.

5. E. Marczewski (Szpilrajn), The characteristic function of a sequence of sets and some of its applications. Fund. Math. 31 (1938), 207-223.

6. E. Marczewski and R. Sikorski, Measures in non-separable metric spaces, Colloq. Math. 1 (1948), $133-139$.

7. D. Scott. Measurable cardinals and constructible sets, Bull. Acad. Polon. Sci. Ser. Sci. Math. Astronom. Phys. 9(1961), 521-524.

8. W. Sierpinski, Sur l'extension des fonctions de Baire définies sur les ensembles linéaires quelconques, Fund. Math. 16 (1930), 31.

9. S. Ulam, Zur Masstheorie in der allgemeinen Mengenlehre, Fund. Math. 16 (1930), 140-150.

Department of Mathematics, Massachusetts institute of Technology, Cambridge, MasSACHUSETTS 02139

Current address: Department of Mathematics, Michigan Technological University, Houghton, Michigan 49931 